On this basis, the locations of the bowel cancers were found to be 19 in the right colon and 68 in the left.

We attempted to corelate clinical findings with site of tumours. It was observed that anaemia was more commonly associated with a right colonic lesion as compared to PR Bleeding which was seen with a left sided pathology.

Abdominal pain and change in bowel habit were not strongly associated with any particular location for a tumour.

Conclusion Our audit data of colon cancers from a community based setting is one of the few to be published in recent years.

We have shown an incidence of about $2 \%$ of colon cancers from this setting.

There appears to be a definite association between PR bleeding and left colon tumours as compared to anaemia which appeared to be associated with right colonic lesions.

The overwhelming majority of cases referred to us were lesions located in the left colon suggesting that one-off flexible sigmoidoscopy as a tool for bowel cancer screening should have a very good diagnostic yield.

Disclosure of Interest None Declared.

\section{PWE-031 HOW MANY COLORECTAL CANCERS (CRC) HAVE BEEN MISSED IN THE 36 MONTHS AFTER 'NORMAL' LOWER GI ENDOSCOPY (COLONOSCOPY/FLEXIBLE SIGMOIDSCOPY)}

doi:10.1136/gutjnl-2013-304907.320

1."K Keshk, 'J T Green, 'G Swift. 'Department of gastroenterology, Cardiff and Vale University Health Board, Cardiff, UK

Introduction Colonoscopy and flexible sigmoidscopy are the primary tools for diagnosis of CRC, although they have false negative rates of $2-6 \% .{ }^{1}$ The aim of this study was to estimate how frequently lower GI endoscopy might have failed to detect cancer within 36 months preceding a confirmed diagnosis of CRC.

Methods We identified 253 patients diagnosed with CRC between Sep 2010 - Aug 2012 from the database of cellular pathology in Cardiff and Vale University Health Board. Medical records were reviewed for the results of colonoscopy, flexible sigmoidscopy, histology and CT imaging. Patients with missed cancer were those who had had a 'normal' (if no cancer discovered) lower GI endoscopy procedure 1-36 months before diagnosis. We examined the characteristics that might be risk factors for missed CRC.

Results Among the 253 patients included in the study, cancer was located in the rectosigmoid colon $(78.5 \%)$, descending colon $(2.7 \%)$, splenic flexure $(1.9 \%)$, transverse colon $(4.3 \%)$, hepatic flexure (1.1\%) and right colon (9.4\%). We identified 10 (5 females, 5 males, mean age 80 years, range $42-92$ ) patients (3.9\%) who had had colonoscopy and/or flexible sigmoidscopy that had not shown CRC 1-36 months prior to the final diagnosis. In the missed cancer group only one flexible sigmoidscopy was incomplete due to suboptimal bowel preparation and difficult sigmoid bend. Nine patients had false negative lower GI endoscopy. The median diagnostic delay was 13.6 months (1-36). Two of the missed cancers were diagnosed with Dukes' C colon cancer, one with Dukes' D colon cancer and one presented with emergency complications due perforation. Of the ten missed cancers, eight were in rectosigmoid colon, one in the transverse colon and one in the ascending colon.

Conclusion Bressler et al reported 2-6\% missed CRC at colonoscopy performed 6-36 months prior to a final diagnosis of cancer. ${ }^{1}$ Our miss rates are in line with previous studies. However, the available literature suggests that lesion miss rate is higher for proximal colonic tumours. In our study, the missed cancers were predominantly in the rectosigmoid colon $(3.1 \%)$ and were minimal in the right colon $(0.4 \%)$. The reasons for missed cancers in our study are likely related to incomplete procedure, suboptimal bowel preparation, inadequate technique, failure to recognise flat lesions and diverticulosis. Optimal withdrawal technique, good luminal view, frequent position change, high quality bowel prep and adequate time for inspection are of utmost importance to minimise the rate of missed CRC.

Disclosure of Interest None Declared.

\section{REFERENCE}

1. Bressler B, et al. Rates of new or missed colorectal cancer after colonoscopy and their risk factors: a population-based study. Gastroenterology 2007; 132(1):96-102

\section{PWE-032 WHAT IS THE IMPACT OF THE INTRODUCTION OF THE NHS BOWEL CANCER SCREENING PROGRAMME ON THE WORKLOAD OF A COLORECTAL SURGERY UNIT AT A DISTRICT GENERAL HOSPITAL?}

doi:10.1136/gutjnl-2013-304907.321

1,"K E Carney, 'R Thomas, 'H Lambson, 'A Agarwal, ${ }^{2} \mathrm{~W}$ Copping, 'D Borowski, 'D Garg, ${ }^{1} \mathrm{~T}$ Gill, ${ }^{\prime} \mathrm{M}$ Tabaqchali. ${ }^{\top}$ Colorectal Surgery; ${ }^{2}$ Bowel Cancer Screening, University Hospital North Tees, Stockton on Tees, UK

Introduction A study to assess the impact of the introduction of the National Bowel Cancer Screening Programme (BCSP) on the workload of a colorectal unit in a medium-size general hospital covering a population of 400,000 , and to compare it to the initially projected figures.

Methods We used the BCSP database, hospital episode statistics and the locally held colorectal cancer Multi-Disciplinary Team (MDT) database to identify all patients with newly diagnosed colorectal cancer diagnosis between April 2007 and November 2012. Demographic data, pathological data, MDT outcomes and treatment details were examined.

Results During the study period of 66 months, a total of 194 patients with screening-detected colorectal cancer were referred to the local MDT (mean 32 cases per annum). Of these, 144 patients had a cancer resection ( $74.2 \%$, mean 24 cases per annum). The remaining 50 cases included unfit patients as well as those with metastatic disease at presentation and patients with polyp cancers not requiring resection. Furthermore, there was an increase in numbers seen from 2010 onwards, coinciding with the increase in the screening age limit to 75 years.

Conclusion This study measures the effect of the national BCSP on the surgical workload and quantifies the proportion of patients requiring surgery. It was projected that 33 people per annum newly diagnosed with colorectal cancer would be referred from the BCSP centre to the local $\mathrm{MDT}^{1}$; this study confirms this projection.

Disclosure of Interest None Declared.

REFERENCE

1. BCSP Publication No. 3 (2008): Guidance for public health and commissioners.

\section{Endoscopy}

\section{PWE-033 THE INCIDENCE AND COST OF UNEXPECTED HOSPITAL ATTENDANCE FOLLOWING ELECTIVE OUTPATIENT FLEXIBLE SIGMOIDOSCOPY}

doi:10.1136/gutjnl-2013-304907.322

1.*H Gordon, 'J H Brindley, 'C Zheng, 'J Graby, 'M A Abdullah, 'J Mawdsley, 'I Beveridge, ${ }^{2} \mathrm{~K}$ Monahan. 'West Middlesex University Hospital, London, UK; ${ }^{2}$ Gastroenterology, West Middlesex University Hospital, London, UK

Introduction Outpatient flexible sigmoidoscopy is an increasingly utilised investigation shown to be effective in the detection and prevention of bowel cancer. The procedure is thought to entail a low risk of complications. However, recent literature suggests the complication rate of other endoscopic procedures may be up to 10 fold 